\title{
Genome-wide mapping of histone modifications during axenic growth in two species of Leptosphaeria maculans showing contrasting genomic organization
}

\author{
Jessica L. Soyer $(\mathbb{D} \cdot$ Colin Clairet $(\mathbb{D})$ Elise J. Gay • \\ Nicolas Lapalu (D) Thierry Rouxel (1) - Eva H. \\ Stukenbrock (1) - Isabelle Fudal (1)
}

Received: 20 November 2020 / Revised: 16 February 2021 / Accepted: 3 March 2021 / Published online: 21 May 2021

(C) The Author(s) 2021

\begin{abstract}
Leptosphaeria maculans 'brassicae' (Lmb) and Leptosphaeria maculans 'lepidii' (Lml) are closely related phytopathogenic species that exhibit a large macrosynteny but contrasting genome structure. Lmb has more than $30 \%$ of repeats clustered in large repeatrich regions, while the Lml genome has only a small amount of evenly distributed repeats. Repeat-rich regions of Lmb are enriched in effector genes, expressed during plant infection. The distinct genome structures of $\mathrm{Lmb}$ and Lml provide an excellent model for comparing the organization of pathogenicity genes in relation to the chromatin landscape in two closely related phytopathogenic fungi. Here, we performed chromatin immunoprecipitation (ChIP) during axenic culture, targeting histone modifications typical for heterochromatin or euchromatin, combined with transcriptomic analysis to analyze the influence of chromatin organization on gene expres-
\end{abstract}

Responsible editor: Andreas Houben

Eva H. Stukenbrock and Isabelle Fudal are co-last authors.

J. L. Soyer $(\bowtie) \cdot$ C. Clairet · E. J. Gay · N. Lapalu •

T. Rouxel $\cdot$ I. Fudal

Université Paris-Saclay, INRAE, AgroParisTech, UMR BIOGER, 78850 Thiverval-Grignon, France

e-mail: jessica.soyer@inrae.fr

J. L. Soyer - E. H. Stukenbrock

Max Planck Institute for Evolutionary Biology,

August-Thienemann-Str. 2, 24306 Plön, Germany

J. L. Soyer - E. H. Stukenbrock

Christian-Albrechts University of Kiel, Am Botanischen Garten 1-9, 24118 Kiel, Germany sion. In both species, we found that facultative heterochromatin is enriched with genes lacking functional annotation, including numerous effector and speciesspecific genes. Notably, orthologous genes located in H3K27me3 domains are enriched with effector genes. Compared to other fungal species, including $\mathrm{Lml}, \mathrm{Lmb}$ is distinct in having large $\mathrm{H} 3 \mathrm{~K} 9 \mathrm{me} 3$ domains associated with repeat-rich regions that contain numerous speciesspecific effector genes. Discovery of these two distinctive heterochromatin landscapes now raises questions about their involvement in the regulation of pathogenicity, the dynamics of these domains during plant infection and the selective advantage to the fungus to host effector genes in $\mathrm{H} 3 \mathrm{~K} 9 \mathrm{me} 3$ or $\mathrm{H} 3 \mathrm{~K} 27 \mathrm{me} 3$ domains.

Keywords Leptosphaeria maculans · ChIP-seq · comparative epigenomics · heterochromatin · effectors · host-pathogen interaction

\begin{tabular}{ll}
\multicolumn{2}{l}{ Abbreviations } \\
bp & $\begin{array}{l}\text { base pair } \\
\text { coding sequence } \\
\text { CDS }\end{array}$ \\
ChIP-seq & $\begin{array}{l}\text { chromatin immunoprecipitation } \\
\text { followed by high-throughput sequencing } \\
\text { false discovery rate }\end{array}$ \\
FDR & $\begin{array}{l}\text { Gene Ontology } \\
\text { GO }\end{array}$ \\
HP1 & $\begin{array}{l}\text { heterochromatin protein } 1 \\
\text { kb }\end{array}$ \\
KMT & $\begin{array}{l}\text { lysine methyltransferase } \\
\text { defective in methylation } \\
\text { DIM }\end{array}$ \\
H3K4me2 & $\begin{array}{l}\text { dimethylation of the lysine } 4 \text { of } \\
\text { histone H3 }\end{array}$
\end{tabular}




$\begin{array}{ll}\text { H3K9me3 } & \begin{array}{l}\text { trimethylation of the lysine } 9 \\ \text { of histone H3 } \\ \text { trimethylation of the lysine } 27 \\ \text { of histone H3 }\end{array} \\ \text { H3K27me3 } & \begin{array}{l}\text { Leptosphaeria maculans 'brassicae' } \\ \text { Lmb }\end{array} \\ \text { Lml } & \begin{array}{l}\text { Leptosphaeria maculans 'lepidii' } \\ \text { non-ribosomal peptide synthetase } \\ \text { NRPS }\end{array} \\ \text { PKS } & \begin{array}{l}\text { polyketide synthase } \\ \text { reads per kilobase per million mapped } \\ \text { RPKM }\end{array} \\ \text { reads } \\ \text { SC } & \begin{array}{l}\text { SuperContig } \\ \text { small secreted protein } \\ \text { transposable element }\end{array} \\ \text { TE } & \end{array}$

\section{Introduction}

Each year, hundreds of millions of tons of agricultural crops are devastated by plant pathogenic fungi or made unfit for consumption due to contamination by mycotoxins (Fisher et al. 2018). Management strategies to control fungal infection mainly involve chemical control or breeding for naturally resistant crop cultivars. However, fungal plant pathogens have proven capable of rapidly evolving resistance against fungicides (Fisher et al. 2018) and to overcome specific plant resistance genes within a few years (for instance in Leptosphaeria maculans; Rouxel and Balesdent 2017), emphasizing the need for improved control methods.

Understanding the determinants of the extreme adaptive abilities of fungal plant pathogens is a critical issue for the development of effective and sustainable control methods. In that respect, comparative and population genomic analyses have provided new insights into the evolutionary dynamics of fungal plant pathogens (e.g. Stukenbrock et al. 2011; Grandaubert et al. 2014; Sánchez-Vallet et al. 2018). Notably, transposable elements (TE) have been shown to play a crucial role in shaping the genome structure of plant pathogenic fungi (Ohm et al. 2012; Grandaubert et al. 2014; Möller and Stukenbrock 2017; Seidl and Thomma 2017). TEs are often organized in clusters, compartmentalizing the genome into gene-rich regions and TE-rich regions (e.g. in L. maculans and in Mycosphaerella fijiensis; Rouxel et al. 2011; Ohm et al. 2012; Grandaubert et al. 2014). While the gene density in TE-rich regions is low, genes located in these regions have been shown to evolve faster than genes located in TE-poor regions (Croll and
McDonald 2012; de Jonge et al. 2013). Interestingly, rapidly evolving genes in TE-rich regions have, in many cases, been identified as genes involved in niche adaptation and notably effector genes. Effectors are considered as key elements of pathogenesis, allowing pathogens to circumvent host recognition, impede defence reactions and facilitate host invasion. Effectors are mainly secreted proteins (called proteinaceous effectors), but can also be secondary metabolites (called metabolic effectors) or small RNAs (Lo Presti et al. 2015; Collemare et al. 2019; Rocafort et al. 2020). Plants have evolved strategies to recognize and counteract effectors, exposing them to a strong selection pressure by the host immune system (Stergiopoulos et al. 2007; Chuma et al. 2011; Rouxel and Balesdent 2017). Indeed, in the course of the coevolution between a pathogen and its host, the host has developed an active immune system allowing the direct or indirect recognition of some effector molecules, to activate defence responses, often involving a local cell death, called the hypersensitive response. Effectors that can be recognized by the host are called avirulence proteins (Jones and Dangl 2006).

Leptosphaeria maculans 'brassicae' (hereinafter referred to as Lmb) belongs to the Dothideomycete class of Ascomycete fungi and is responsible for causing stem canker of oilseed rape (Brassica napus). Lmb displays a complex, hemibiotrophic life cycle, during which it alternates between different nutritional modes on its host plant. It causes necrosis on different plant organs: leaves, more rarely seedpods and the base of the stem, causing lodging of the plant and yield losses (Rouxel and Balesdent 2005). The most efficient method of disease control relies on the use of major resistance genes present in oilseed rape and other Brassica species. Although efficient, this control method is not sustainable, as Lmb is able to 'break down' novel sources of genetic resistance rapidly (Rouxel and Balesdent 2017). One-third of the Lmb genome is made of TE-rich regions. These regions are enriched with putative effector genes and include all currently known avirulence genes. These avirulence genes are highly expressed in the first 7 days of leaf and cotyledon infection (Gout et al. 2006; Fudal et al. 2007; Parlange et al. 2009; Balesdent et al. 2013; Van de Wouw et al. 2014; Ghanbarnia et al. 2015, 2018; Plissonneau et al. 2016, 2018; Petit-Houdenot et al. 2019; Neik et al. 2020). Another set of putative proteinaceous effector genes are located in gene-rich regions of the genome and are specifically expressed during stem infection (Gervais et al. 2017). 
In eukaryotic cells, chromatin can adopt different conformational states directly influencing gene expression: gene-rich euchromatin, sheltering constitutively expressed genes, and gene-poor heterochromatin, in which genes are silent. The different chromatin states are characterized by different post-translational modifications of histones around which DNA is wrapped. Typically, heterochromatin is enriched in the trimethylation of the lysine 9 of histone $\mathrm{H} 3$ (H3K9me3) and lysine 27 (H3K27me3) while euchromatin is enriched in the di- (or tri-) methylation of the lysine 4 of histone H3 (H3K4me2); (Mikkelsen et al. 2007; Jamieson et al. 2013). In fungal plant pathogens or endophytes, evidence is accumulating that transcriptional reprogramming of effector genes (either proteinaceous or metabolic) is tightly controlled by chromatinbased regulatory mechanisms (for example in Fusarium graminearum, Epichloe festucae, Lmb and Zymoseptoria tritici; Connolly et al. 2013; Chujo and Scott 2014; Soyer et al. 2014, 2015a, 2019; Lukito et al. 2020; Meile et al. 2020). In Lmb, the location of avirulence genes in TE-rich regions has an influence on their evolution under selection pressure (Rouxel et al. 2011; Daverdin et al. 2012) and plays a role in regulating their expression during axenic culture via deposition of H3K9me3 (Soyer et al. 2014).

Lmb belongs to the L. maculans/Leptosphaeria biglobosa species complex, comprising species with different host specialization and genome organization (Grandaubert et al. 2014). Within the species complex, the L. maculans species infecting oilseed rape, Lmb, is the only one having large regions of its genome enriched with TEs while other genomes have a low TE content ( $\sim 3-15 \%$ compared to more than $30 \%$ TEs in Lmb; Rouxel et al. 2011; Grandaubert et al. 2014; Dutreux et al. 2018). For example, the genome of the species that is most closely related to Lmb, Leptosphaeria maculans 'lepidii' (hereinafter referred to as Lml), which infects crucifers such as Lepidium sativum, and to a lesser extent Camelina sativa and Brassica rapa (Petrie 1969), has a low TE content (3\% of TEs; Grandaubert et al. 2014). Interestingly, the genomes of Lmb and Lml show a high level of macrosynteny, with only a few intra-chromosomal inversions, but differ in their TE content with Lmb having undergone a massive TE expansion 5 million years ago corresponding to the speciation date (Grandaubert et al. 2014). Invasion of TEs in the genome of Lmb has shaped its genome, with alternating TE-rich regions and gene-rich regions. In contrast, the Lml genome shows a homogeneous TE distribution along the chromosomes (Grandaubert et al. 2014).

The distinct genome organization shown by Lmb and Lml thus provides us with a model of choice for comparing epigenomic organization in two closely related phytopathogenic fungi, to determine the genomic location of pathogenicity/effector genes in relation to the chromatin landscape and the influence of chromatin structure on gene expression. Comparative epigenomic analyses, at the intra- or inter-species levels, are very sparse and this remains an underexplored field of study, at least in fungi (Zhang et al. 2018; Feurtey et al. 2019). We present here the first comparative epigenomic analysis of both euchromatin and heterochromatin marks in two closely related phytopathogenic fungi. We first performed ChIP-seq and RNA-seq during axenic culture to compare the distribution of three histone modifications, H3K4me2, H3K9me3 and H3K27me3 and then investigated whether chromatin organization is conserved between Lmb and Lml by assessing whether orthologous genes, and pathogenicity-related genes, are located in similar chromatin domains in each species. Lastly, we assessed the influence of the chromatin landscape on gene expression during axenic growth, focusing mostly on pathogenicity-related genes that may be species specific.

\section{Materials and methods}

Fungal isolates

The isolate v23.1.3 of L. maculans 'brassicae' and the isolate IBCN84 of L. maculans 'lepidii' were used throughout the analyses (Rouxel et al. 2011; Grandaubert et al. 2014). Fungal cultures were maintained as described previously (Ansan-Melayah et al. 1995). For chromatin immunoprecipitation and transcriptomic analyses performed during in vitro growth, mycelium of $\mathrm{Lmb}$ and $\mathrm{Lml}$ were grown on $\mathrm{V} 8$ agar medium at $25{ }^{\circ} \mathrm{C}$ for 7 days. Then 10 plugs of mycelium were inoculated into $100 \mathrm{ml}$ of Fries liquid medium in a Roux bottle. Mycelia were harvested after growing for 7 days at $25^{\circ} \mathrm{C}$, filtered and washed thoroughly with distilled water and immediately placed in liquid nitrogen until further used. ChIP experiments were performed on fresh material. 
Chromatin immunoprecipitation and high-throughput sequencing

ChIP was performed from freshly harvested mycelium grown in Fries liquid culture, as described in Soyer et al. (2015b), with minor modifications. ChIP was performed on native material (without crosslinking) using antibodies targeting histone modifications H3K4me2 (Merck ref. 07-030), H3K9me3 (Active Motif, Carlsbad, CA, USA; ref. 39,161) or H3K27me3 (Active Motif, Carlsbad, CA, USA; ref. 39155). Three different ChIPs (i.e. three biological replicates) were performed for each of the histone modifications. Libraries were prepared from all biological replicates, individually, according to the Illumina TruSeq protocol 'Ultra Low Input DNA library'. Libraries were sequenced with an Illumina HiSeq 2000 genome analyzer at the Max Planck Genome Centre Cologne, Germany (https://mpgc.mpipz. mpg.de/home/). Sequencing data are available under the GEO accession number GSE150127.

Analysis of ChIP-seq data and identification of significantly enriched domains

Analysis of ChIP-seq datasets was performed as described in Schotanus et al. (2015). Quality of Illumina reads was analyzed using FastQC (https://www. bioinformatics.babraham.ac.uk/projects/fastqc/). Based on results of this analysis, $10 \mathrm{bp}$ were trimmed from the $5^{\prime}$ end. Processed reads were mapped on the reference genome of Lmb (Dutreux et al. 2018) or Lml (Grandaubert et al. 2014) using Bowtie2 (Langmead and Salzberg 2012) with default parameters (Supplementary Table 1). Peak calling analysis was performed on each ChIP sequencing dataset, to identify significantly enriched domains for either H3K4me2, H3 K9me3 or H3K27me3, using RSEG (Song and Smith 2011). A domain was considered if identified in at least two out of the three biological replicates. The Integrative Genome Viewer (Thorvaldsdóttir et al. 2013) was used to visualize location of each domain along genomes of $\mathrm{Lmb}$ and $\mathrm{Lml}$ according to other genome features (gene annotation, TE annotation, GC content). Coverage of the histone modifications was assessed in 10-kb nonoverlapping sliding windows along the supercontigs and correlation analyses, using Kendall's $T$ correlation coefficient, were performed between biological replicates to check for reproducibility (Supplementary Tables 2 and 3).
RNA extraction, RNA-sequencing and expression analysis

Total RNA was extracted from mycelium grown for 1 week in Fries liquid medium as previously described (Fudal et al. 2007). The NEBNext Ultra Directional RNA Library Prep Kit for Illumina (cat. \# E7420L New England BioLabs) was used to prepare RNA-seq libraries and sequencing was performed on a HiSeq 4000 Illumina genome analyzer using 50 paired-end reads. Raw reads were then pre-processed with Trimmomatic (Bolger et al. 2014) to remove short reads ( $<30$ bp) and eliminate sequencing adaptors. Cleaned reads were then mapped against each genome using STAR with default parameters (Dobin and Gingeras 2015; Supplementary Table 1). Gene expression was evaluated using the rpkm_count function of EdgeR (Robinson et al. 2010). Genes with RPKM $\geq 2$ were considered expressed. Sequencing data are available under the GEO accession number GSE150127.

Identification of orthologues and annotation of genes encoding proteinaceous effectors in $\mathrm{Lmb}$ and $\mathrm{Lml}$

The genome of Lmb encodes 13,047 proteins and that of Lml 11,272 proteins (Grandaubert et al. 2014; Dutreux et al. 2018). Orthologous proteins between Lmb and Lml were identified using OrthoFinder with default parameters (Emms and Kelly 2015).

Based on the new assembly and annotation available for the genome of Lmb (Dutreux et al. 2018), an updated repertoire of putative proteinaceous effector genes (encoding Small Secreted Proteins, SSP) has been predicted. Therefore, to compare effector repertoires of $\mathrm{Lmb}$ and $\mathrm{Lml}$, the same pipeline for the prediction of putative effector genes was applied to both species. Signal peptide and subcellular localization were predicted by SignalP version 4.1 (Petersen et al. 2011) and TargetP version 1.1 (Almagro Armenteros et al. 2019) respectively. Transmembrane domains were predicted by TMHMM version 2.0. The predicted secretome contained all proteins with no more than one transmembrane domain and either a predicted signal peptide or a predicted extracellular localization. The final effector repertoires were created by applying a size cutoff of 300 amino acids on the predicted secretome. In parallel, EffectorP version 1.0 (Sperschneider et al. 2016) was used on the predicted secretome, and for $\mathrm{Lml}$, four proteins were predicted as effectors with a size higher 
than 300 amino acids. These four proteins were added to the SSP set. This predicted a repertoire of 1080 and 892 $\mathrm{SSP}$-encoding genes for $\mathrm{Lmb}$ and for $\mathrm{Lml}$, respectively.

Analysis of GO enrichment and statistical analyses

Gene Ontology (GO) annotations of the Lmb and Lml genes were retrieved from Dutreux et al. (2018) and Grandaubert et al. (2014) respectively. GO term enrichment analysis of the H3K4me2-, H3K9me3- and H3K27me3-associated genes was performed with the plug-in Biological networks Gene ontology (BinGo; v3.0.3) of the cytoscape software (Shannon et al. 2003). List of genes submitted to BINGO were considered as significantly enriched for a given GO term with an associated false discovery rate (FDR) $\leq 0.01$ for the biological processes.

In order to assess significant enrichment of $\mathrm{H} 3 \mathrm{~K} 4 \mathrm{me} 2$, H3K9me3 or H3K27me3 in certain categories of genes (such as proteinaceous or metabolic effector-encoding genes), a Chi ${ }^{2}$ test, or a Fisher's exact test for small sized-population (Kim 2017), was applied to compare the expected and observed proportions of genes of a given category in $\mathrm{H} 3 \mathrm{~K} 4 \mathrm{me} 2$, $\mathrm{H} 3 \mathrm{~K} 9 \mathrm{me} 3$ or H3K27me3 domains (Soyer et al. 2019). Enrichment was considered significant with a $P<0.01$. A Wilcoxon test was applied to identify significant differences in terms of the expression of genes associated with $\mathrm{H} 3 \mathrm{~K} 4 \mathrm{me} 2$, $\mathrm{H} 3 \mathrm{~K} 9 \mathrm{me} 3$ and H3K27me3 during axenic culture (considered significant with a $P<0.01)$. Analyses were done using R, version 3.0.2 (www.r-project.org).

\section{Results}

A different genome organization but similar epigenomic properties in both genomes

To assess the genome-wide distribution of histone marks in Lmb and Lml during axenic growth, we performed ChIP-seq experiments using antibodies against histone modifications $\mathrm{H} 3 \mathrm{~K} 4 \mathrm{me} 2, \mathrm{H} 3 \mathrm{~K} 9 \mathrm{me} 3$ and H3K27me3 (Supplementary Table 1). Mapping of the ChIP-seq data was followed by identification of significantly enriched domains, for any of the histone modifications targeted, and reproducibility of the biological replicates was assessed (Supplementary Tables 2 and 3). Based on the genomic coordinates of the H3K4me2, $\mathrm{H} 3 \mathrm{~K} 9 \mathrm{me} 3$ and $\mathrm{H} 3 \mathrm{~K} 27 \mathrm{me} 3$ domains, the number of bases associated with any of the three histone modifications was evaluated, for each genome, in 10-kb sliding windows. In the genomes of $\mathrm{Lmb}$ and $\mathrm{Lml}$, the proportion of $\mathrm{H} 3 \mathrm{~K} 4 \mathrm{me} 2$ and $\mathrm{H} 3 \mathrm{~K} 27 \mathrm{me} 3$ was similar (H3K4me2: 39\% in Lmb vs. 32\% in Lml; H3K27me3: $19 \%$ in $\mathrm{Lmb}$ vs. $13 \%$ in $\mathrm{Lml}$ ) while the proportion of H3K9me3 was strikingly different $(33 \%$ and $4 \%$ for Lmb and Lml, respectively) (Supplementary Tables 4 and 5). The average size of H3K4me 2 and H3K9me3 domains were longer in $\mathrm{Lmb}$ than in $\mathrm{Lml}$ (H3K4me2: 2989 bp in Lmb vs. 1460 bp in Lml; H3K9me3 domains: 8443 bp in Lmb vs. 3499 bp in Lml). Notably, the Lmb genome displayed exceptionally large H3K9me3 domains, whereby one domain comprised up to $230 \mathrm{~kb}$. In contrast, maximum size of $\mathrm{H} 3 \mathrm{~K} 9 \mathrm{me} 3$ domains in Lml was only $12 \mathrm{~kb}$. However, H3K27me3 domains were on average longer in the $\mathrm{Lml}$ than in the Lmb genome (1500 bp in Lmb vs. 2108 bp in Lml) (Figs. 1, 2, 3, and 4).

In the genomes of $\mathrm{Lmb}$ and $\mathrm{Lml}, \mathrm{H} 3 \mathrm{~K} 4 \mathrm{me} 2$ and H3K9me3 domains were mutually exclusive (Kendall's T: -0.57 and $-0.16, P<2.2 .10^{-16}$ for $\mathrm{Lmb}$ and $\mathrm{Lml}$ respectively; Figs. 2, 3, and 4; Supplementary Figure 1; Tables 1 and 2). We identified a positive correlation between the location of TEs and H3K9me3 domains (Kendall's T: 0.87 and $0.68, P<2.2 .10^{-16}$ for Lmb and Lml respectively; Figs. 2, 3, and 4; Supplementary Figure 1; Tables 1 and 2) and between the location of coding sequences (CDS) and H3K4me2 (Kendall's T: 0.55 and $0.25, P<2.2 .10^{-16}$ for $\mathrm{Lmb}$ and $\mathrm{Lml}$ respectively; Figs. 2, 3, and 4; Supplementary Figure 1; Tables 1 and 2). In the genome of Lmb, a weak correlation was demonstrated between CDS and H3K27me3 domains (Kendall's T: 0.12, $P<2.2 .10^{-16}$; Supplementary Figure 1; Table 1). For Lmb and Lml, the coverage of H3K9me3 and H3K27me3 was not homogeneous across all SCs because some are more than two fold enriched with these histone modifications compared to others (Supplementary Tables 4 and 5; Figs. 3 and 4). An extreme example of this pattern was identified on the dispensable chromosome of Lmb (i.e. SuperContig19; Leclair et al. 1996; Rouxel et al. 2011), which is strongly enriched in TEs compared to the rest of the genome (35\% of TEs in the core genome and $93 \%$ of TEs in the dispensable chromosome). Consistently, we found a strong enrichment in $\mathrm{H} 3 \mathrm{~K} 9 \mathrm{me} 3$ on the dispensable chromosome (32\% in the core genome and $90 \%$ in the dispensable chromosome; Supplementary Table 4; Fig. 3), with only a few H3K4me2 and H3K27me3 domains 


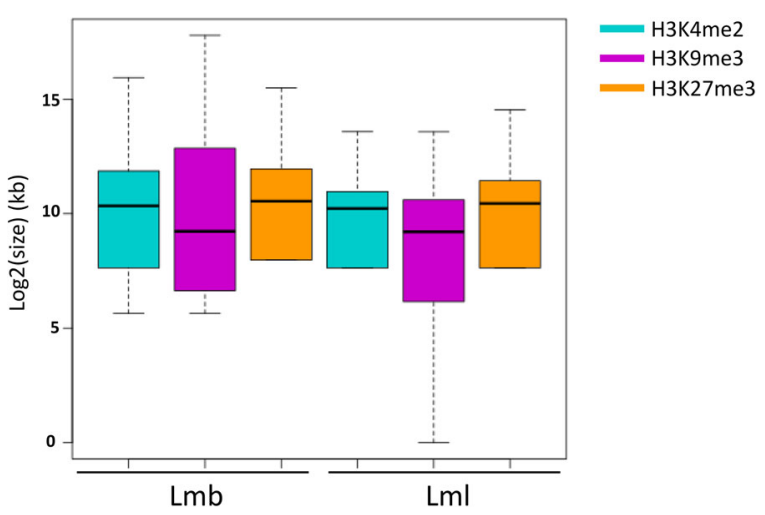

Fig. 1 Size of the histone domains in the genomes of Leptosphaeria maculans 'brassicae' and Leptosphaeria maculans 'lepidii'. $\log 2$ of the size of the domains was estimated based on the coordinates of the location of the domains, identified using RSEG (Song and Smith 2011). Blue: H3K4me2; purple: H3K9me3; Orange: H3K27me3; Lmb, L. maculans 'brassicae'; Lml, L. maculans 'lepidii'

(Fig. 3). In Lmb, we found that many sub-telomeric regions showed overlaps between $\mathrm{H} 3 \mathrm{~K} 9 \mathrm{me} 3$ and H3K27me3, whereas these marks did not overlap on the chromosome arms, even within large TE-rich regions (Fig. 3). The assembly quality of the Lml genome however did not allow us to evaluate whether this was a common feature between the two species.

H3K4me2 domains associate with genes involved in primary metabolism while $\mathrm{H} 3 \mathrm{~K} 27 \mathrm{me} 3$ domains shelter genes involved in niche adaptation and cell wall degradation

Genes associated with $\mathrm{H} 3 \mathrm{~K} 4 \mathrm{me} 2, \mathrm{H} 3 \mathrm{~K} 9 \mathrm{me} 3$ or H3K27me3 domains were identified in the genomes of $\mathrm{Lmb}$ and $\mathrm{Lml}$ during axenic growth. Overall, the number of genes associated with any of the histone modifications was similar in both species, with more than $50 \%$ of the predicted genes associated with $\mathrm{H} 3 \mathrm{~K} 4 \mathrm{me} 2, \sim 14 \%$ of the genes associated with $\mathrm{H} 3 \mathrm{~K} 27 \mathrm{me} 3$ and only a few genes associated with H3K9me3 (104 and 70 respectively in $\mathrm{Lmb}$ and $\mathrm{Lml}$; Fig. 5). In $\mathrm{Lmb}$, there was a higher number of genes associated with both H3K4me2 and H3K27me3 than in Lml (2044 and 361 for Lmb and Lml, respectively) (Fig. 5). For both genomes, we could assign 30-40\% of the predicted genes to a GO category (5076 and 3725 genes for Lmb and Lml, respectively; Grandaubert et al. 2014; Dutreux et al. 2018). We found that H3K4me2 was enriched with genes with a GO annotation $\left(X^{2}\right.$ test, $P<$
2.2.10 $0^{-16}$ ) because more than $40 \%$ of these genes had a GO annotation (3276 and 2581 genes for Lmb and Lml respectively). Between 20 and $28 \%$ of the genes associated with H3K27me3 (572 and 288 genes for $\mathrm{Lmb}$ and $\mathrm{Lml}$, respectively) had a GO annotation. However, none of the genes located in $\mathrm{H} 3 \mathrm{~K} 9 \mathrm{me} 3$ domains, H3K9/K27me3 domains or H3K4me2/H3K27me3 domains had a GO annotation. In other words, genes located in heterochromatin were enriched with genes without a known function $\left(X^{2}\right.$ test, $\left.P<2 \cdot 2 \cdot 10^{-16}\right)$. For both species, genes associated with $\mathrm{H} 3 \mathrm{~K} 4 \mathrm{me} 2$ displayed a wide variety of annotated functions corresponding to primary metabolism and basic cellular functions, such as translation (GO:0006412; 206 genes) or cellular protein metabolic process (Supplementary Tables 6, 7). For both species, only a few GO terms were identified among genes associated with H3K27me3. Nevertheless, in Lmb, these genes were significantly enriched in GO terms associated with carbohydrate metabolic process (GO:0005975; 82 genes), oxydo-reduction process (GO:0055114; 149 genes) and transmembrane transport (GO:0055085; 104 genes) $(P \leq 0.01)$ (Supplementary Table 8 ). As for $\mathrm{Lml}$, probably due to the lower number of genes associated to $\mathrm{H} 3 \mathrm{~K} 27 \mathrm{me} 3$, only a few GO annotations were detected. We only identified one GO category that was found in common with Lmb, namely carbohydrate metabolic process (GO:0005975; 41 genes). Other enrichments corresponded to a few genes classified as response to chemical (GO: 42221; 20 genes), response to nitrogen compound (GO:1901698; 10 genes), response to organophosphorus (GO: 46683, 10 genes) and others (Supplementary Table 9). Although most GO terms enriched among genes associated with H3K27me3 in Lmb and Lml did not overlap, GO terms identified suggest that $\mathrm{H} 3 \mathrm{~K} 27 \mathrm{me} 3$-associated genes might not only be involved in stress response mechanisms, but may also be involved in feeding or cell wall degradation processes during plant infection.

H3K4me2 domains are associated with expressed genes while $\mathrm{H} 3 \mathrm{~K} 9 \mathrm{me} 3$ and $\mathrm{H} 3 \mathrm{~K} 27 \mathrm{me} 3$ domains are associated with silent genes during axenic growth

We performed a genome-wide transcriptome profiling of fungal cultures grown in vitro, and we correlated gene expression patterns with the distribution of histone modifications. In Lmb, 10,934 genes (83\% of the predicted genes) and 7735 genes in Lml (69\% of the predicted genes) were expressed during vegetative growth. 


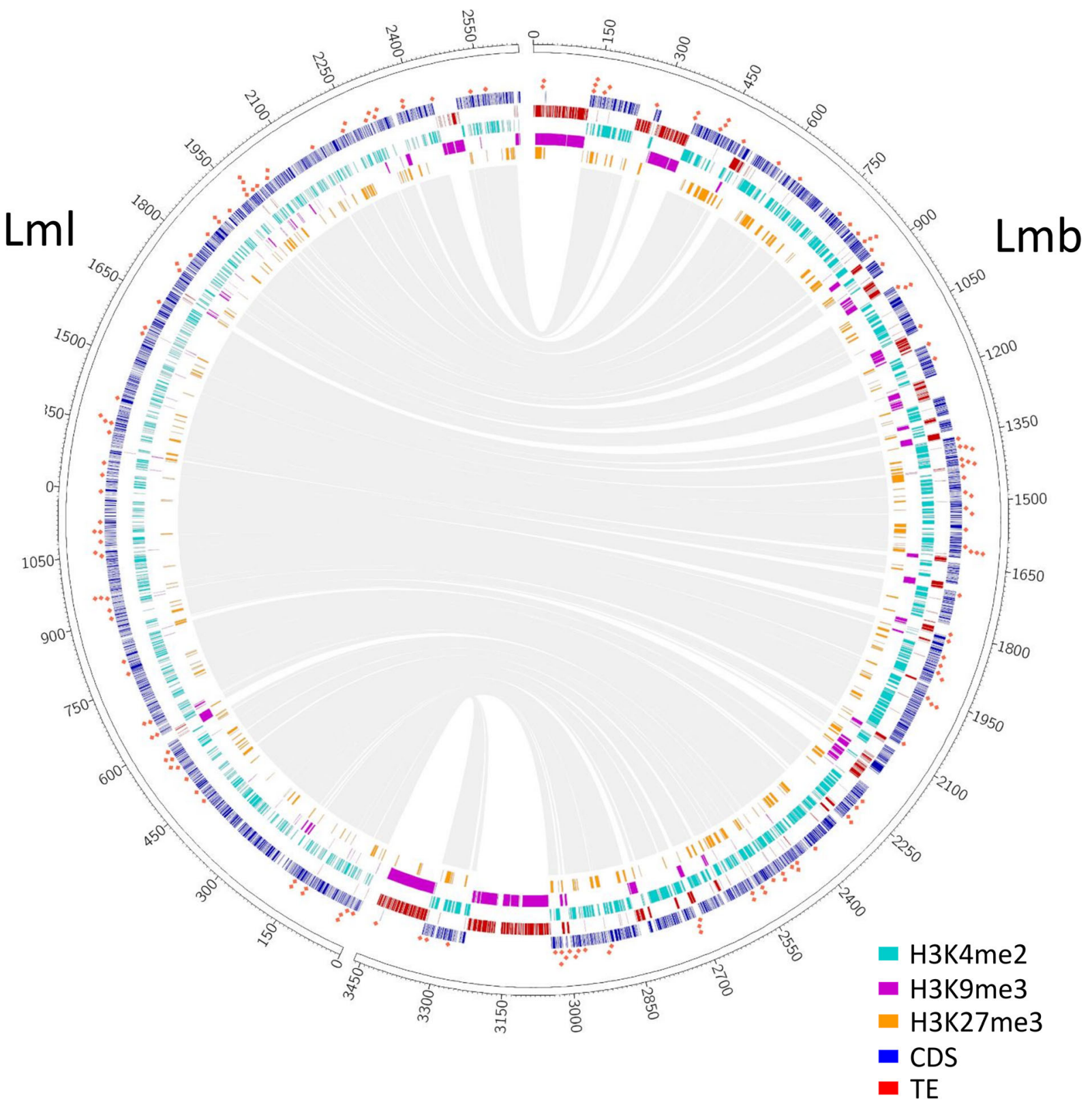

Fig. 2 Genome of Leptosphaeria maculans 'brassicae' harbours large TE-rich, H3K9me3 domains compared to Leptosphaeria maculans 'lepidii'. Example of SuperContig 2 of Lmb and Scaffold 1 of Lml. ChIP-seq was performed with antibodies targeting H3K4me2 (light blue), H3K9me3 (purple) or H3K27me3 (orange); rectangles indicate location of significantly enriched domains identified using RSEG (Song and Smith 2011). Location of CDS (dark blue); transposable elements (TE, red); H3K4me2 (light blue); H3K9me3 (purple); H3K27me3 (orange); Lmb, L. maculans 'brassicae'; Lml, L. maculans 'lepidii'. Genes encoding proteinaceous effectors are indicated with a red square
Considering the top 100 most expressed genes of Lmb, 68 were associated with $\mathrm{H} 3 \mathrm{~K} 4 \mathrm{me} 2$ while two were located within a $\mathrm{H} 3 \mathrm{~K} 27 \mathrm{me} 3$ domain and none in a
H3K9me3 domain (data not shown). In Lml, 71 were located within a $\mathrm{H} 3 \mathrm{~K} 4 \mathrm{me} 2$ domain while five were located in a H3K27me3 domain. These data were 


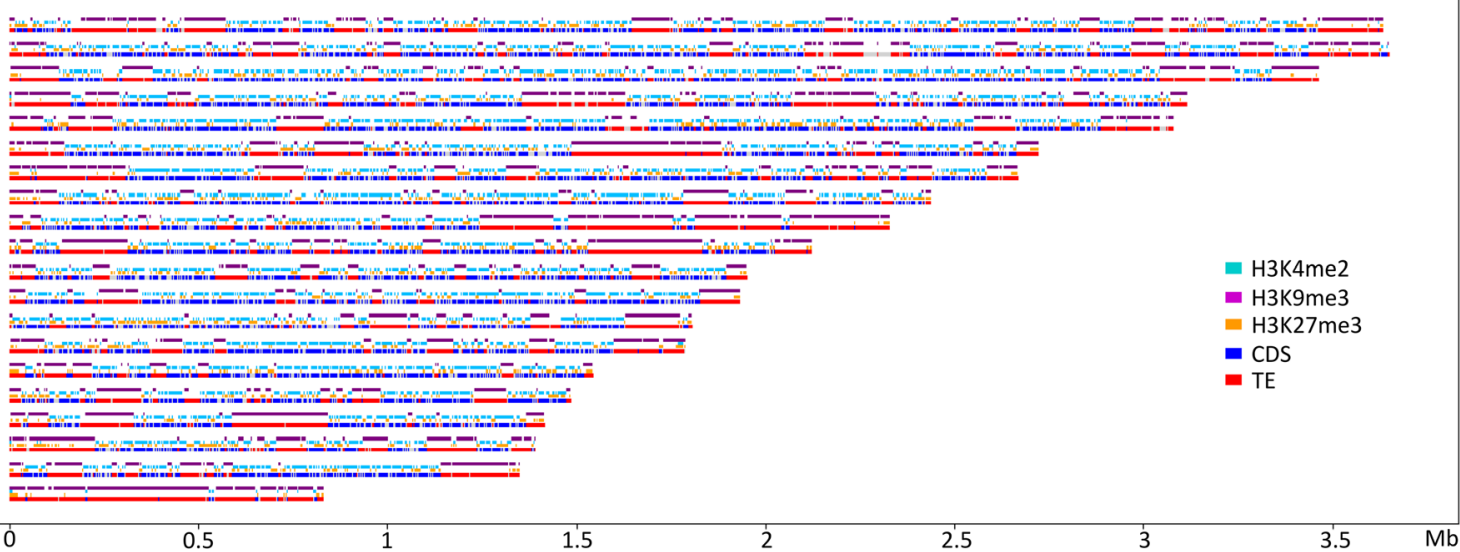

Fig. 3 Location of different genome features in the genome of Leptosphaeria maculans 'brassicae'. SuperContig (SC) 0 to 19 are being the dispensable chromosome. Location of CDS (dark blue); transposable elements (TE, red); H3K4me2 domains (light blue); displayed, representing 97\% of the Lmb genome, with SC 19 H3K9me3 domains (purple); H3K27me3 domains (orange)

confirmed at a genome-wide scale because H3K4me2 domains were enriched with genes expressed during axenic culture (7104 of the genes associated with H3K4me2 were expressed in Lmb and 5338 in Lml; Fig. 6; $P<2.2 .10^{-16}$ ). On the contrary, H3K9me3 and H3K27me3 domains were enriched with genes that were silent during in vitro growth (Fig. 6; $P<$ 2.2.10 $\left.{ }^{-16}\right)$. On the other hand, in both species, genes that were associated with both $\mathrm{H} 3 \mathrm{~K} 4 \mathrm{me} 2$ and H3K27me3 were expressed during axenic growth (90\% and $87 \%$ of the genes, respectively, in Lmb and $\mathrm{Lml}$ ) and their level of expression was similar to that of genes located in $\mathrm{H} 3 \mathrm{~K} 4 \mathrm{me} 2$ domains (Fig. 6). We also investigated the influence of $\mathrm{H} 3 \mathrm{~K} 4 \mathrm{me} 2$, $\mathrm{H} 3 \mathrm{~K} 9 \mathrm{me} 3$ or H3K27me3 on expression of genes encoding proteinaceous effectors and observed that these histone

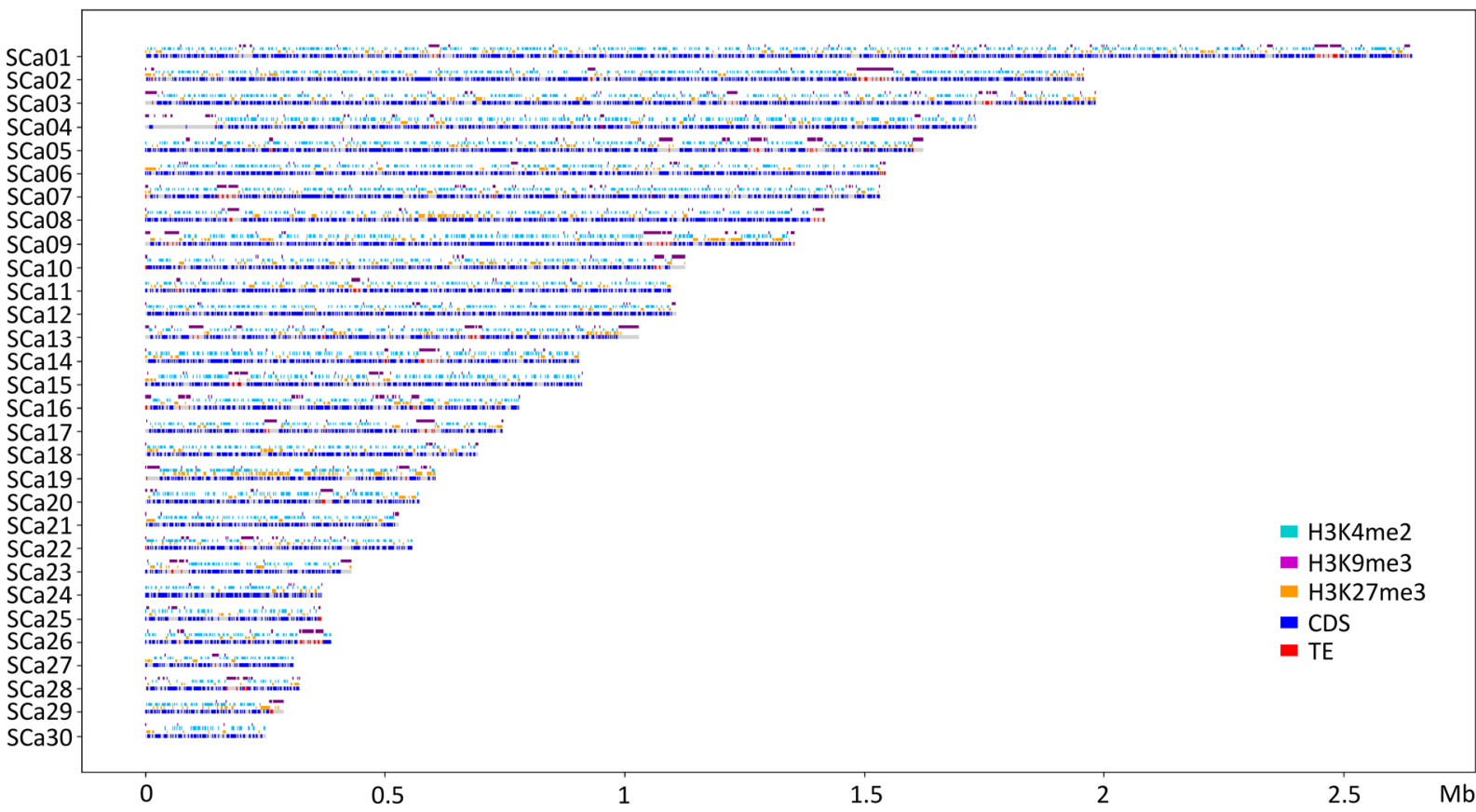

Fig. 4 Location of different genome features in the genome of Leptosphaeria maculans 'lepidii'. Scafflods (SCa) 1 to 30 are displayed, representing $93 \%$ of the $\mathrm{Lml}$ genome. Location of
CDS (dark blue); transposable elements (TE, red); H3K4me2 domains (light blue); H3K9me3 domains (purple); H3K27me3 domains (orange) 
Table 1 Correlation between transposable elements, coding sequences and histone modifications in the Leptosphaeria maculans 'brassicae' genome

\begin{tabular}{llllll}
\hline & CDS & TE & H3K4me2 & H3K9me3 & H3K27me3 \\
\hline CDS & 1.00 & -0.63 & 0.55 & -0.60 & 0.12 \\
TE & -0.63 & 1.00 & -0.58 & 0.87 & -0.25 \\
H3K4me2 & 0.55 & -0.58 & 1.00 & -0.57 & -0.11 \\
H3K9me3 & -0.60 & 0.87 & -0.57 & 1.00 & -0.24 \\
H3K27me3 & 0.12 & -0.25 & -0.11 & -0.24 & 1.00 \\
\hline
\end{tabular}

The coverage of transposable elements (TE), coding sequences (CDS) and histone modifications (H3K9me3, H3K27me3, H3K4me2) along the genome of L. maculans 'brassicae' was analyzed in 1000-bp sliding windows. A Kendall's T correlation analysis was done using $\mathrm{R}$

modifications have the same effect on expression of effector genes as for any other genes (Supplementary Figure 2). We further assessed whether the extent to which a gene is associated with $\mathrm{H} 3 \mathrm{~K} 4 \mathrm{me} 2, \mathrm{H} 3 \mathrm{~K} 9 \mathrm{me} 3$ or H3K27me3 could influence the expression of the associated gene. We classified the genes in different categories based on their association with a particular histone modification and assessed whether this was correlated to the pattern of gene expression (Supplementary Figure 3). Overall, considering different types of coverage (either in the beginning or the end of the CDS, or a coverage of the entire gene), we found that $\mathrm{H} 3 \mathrm{~K} 4 \mathrm{me} 2$ was associated with transcriptional activity while $\mathrm{H} 3 \mathrm{~K} 9 \mathrm{me} 3$ and $\mathrm{H} 3 \mathrm{~K} 27 \mathrm{me} 3$ were associated with transcriptional repression (Supplementary Figure $3 ; P<0.01)$. In Lmb, genes entirely associated with $\mathrm{H} 3 \mathrm{~K} 4 \mathrm{me} 2$ showed the highest level of expression

Table 2 Correlation between transposable elements, coding sequences and histone modifications in the Leptosphaeria maculans 'lepidii' genome

\begin{tabular}{llllll}
\hline & CDS & TE & H3K4me2 & H3K9me3 & H3K27me3 \\
\hline CDS & 1.00 & -0.22 & 0.25 & -0.25 & -0.05 \\
TE & -0.22 & 1.00 & -0.16 & 0.68 & 0.00 \\
H3K4me2 & 0.25 & -0.16 & 1.00 & -0.16 & -0.23 \\
H3K9me3 & -0.25 & 0.68 & -0.16 & 1.00 & 0.01 \\
H3K27me3 & -0.05 & 0.00 & -0.23 & 0.01 & 1.00 \\
\hline
\end{tabular}

The coverage of transposable elements (TE), coding sequences (CDS) and histone modifications (H3K9me3, H3K27me3, $\mathrm{H} 3 \mathrm{~K} 4 \mathrm{me} 2$ ) along the genome of $L$. maculans 'lepidii' was analyzed in 1000-bp sliding windows. A Kendall's $T$ correlation analysis was done using $\mathrm{R}$ while genes entirely associated with $\mathrm{H} 3 \mathrm{~K} 9 \mathrm{me} 3$ or H3K27me3 showed less expression. Genes associated with $\mathrm{H} 3 \mathrm{~K} 9 \mathrm{me} 3$ and $\mathrm{H} 3 \mathrm{~K} 27 \mathrm{me} 3$ grouped together in terms of expression (Supplementary Figure 3; $P<$ 0.01 ). In $\mathrm{Lml}$, genes partially associated with H3K4me2 (either on the beginning or the end of the $\mathrm{CDS}$ ) were more expressed than genes entirely associated with H3K4me2 (Supplementary Figure 3; $P<$ $0.01)$. Contrary to Lmb, the less expressed genes of Lml were those associated with H3K27me3 (Supplementary Figure 3; $P<0.01$ ), which might reflect that a very little amount of $\mathrm{H} 3 \mathrm{~K} 9 \mathrm{me} 3$ is present in the genome of Lml. Thus, our analyses provide evidence that $\mathrm{H} 3 \mathrm{~K} 27 \mathrm{me} 3$ might be the most important regulator of gene expression in the Lml genome. In both species, this transcriptomic analysis confirmed that genes located in a $\mathrm{H} 3 \mathrm{~K} 4 \mathrm{me} 2$ domain were more likely to be expressed while those located in $\mathrm{H} 3 \mathrm{~K} 9 \mathrm{me} 3$ and H3K27me3 domains were more likely to be silent.

Heterochromatin domains are enriched with species-specific genes

A total of 7393 genes were conserved between both species. H3K4me2 domains were enriched with genes conserved between these two species, for example, 4892 $\mathrm{Lmb}$ genes located in euchromatin regions during growth in vitro were conserved $\left(X^{2}\right.$ test, $P<2.2 .10^{-16}$ ) and the vast majority of these (4298 i.e. $88 \%$ ) were expressed during axenic growth in both $\mathrm{Lmb}$ and $\mathrm{Lml}$. Most of the conserved genes associated with H3K4me2 were involved in primary metabolism as mentioned above. Genes associated with both $\mathrm{H} 3 \mathrm{~K} 4 \mathrm{me} 2$ and H3K27me3 were also enriched with conserved genes (1318 and 262 genes, respectively, in Lmb and $\mathrm{Lml}$ ) representing more than $65 \%$ of the genes in these domains ( $X^{2}$ test, $P<5.2 .10^{-3}$ ). In Lmb, two genes involved in heterochromatin assembly and maintenance were analyzed previously through gene silencing (LmHP1 and LmKMT1/DIM5; Soyer et al. 2014). $L m H P 1$ is conserved in $\mathrm{Lml}$ and located in euchromatin in both species. LmKMT1 is also conserved in both species, being located in a $\mathrm{H} 3 \mathrm{~K} 4 \mathrm{me} 2 / \mathrm{H} 3 \mathrm{~K} 27 \mathrm{me} 3$ domain in $\mathrm{Lmb}$ and within a H3K4me2/H3K9me3 domain in $\mathrm{Lml}$, during axenic growth. In contrast, H3K27me3 and $\mathrm{H} 3 \mathrm{~K} 9 \mathrm{me} 3$ domains were significantly enriched with species-specific genes (a total of 865 genes and 661 genes, respectively, in Lmb and Lml; $X^{2}$ test, $P<$ 2.2.10 ${ }^{-16}$ ). Among genes located in heterochromatin in 
a.

L. maculans 'brassicae'

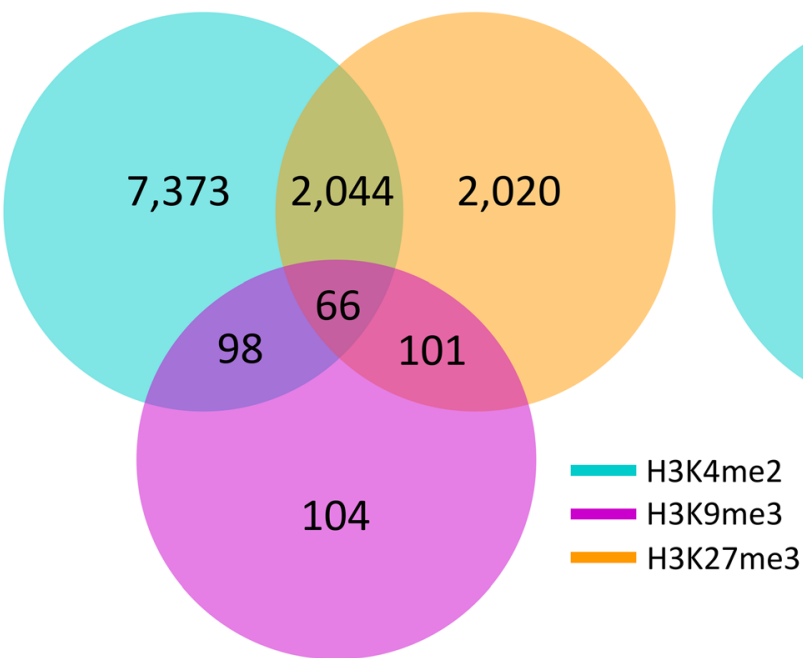

L. maculans 'lepidii'

b.
Fig. 5 Number of genes associated with histone modifications in Leptosphaeria maculans 'brassicae' and Leptosphaeria maculans 'lepidii'. (a) L. maculans 'brassicae', Lmb and (b) L. maculans 'lepidii', Lml. Locations of histone modifications in the genomes of Lmb and Lml were identified using RSEG (Song and Smith

both species (either H3K9me3, H3K27me3 or H3K9/ K27me3 domains), 445 were conserved between Lmb and $\mathrm{Lml}$, of which 83 were expressed and 182 were repressed during axenic growth in both species.

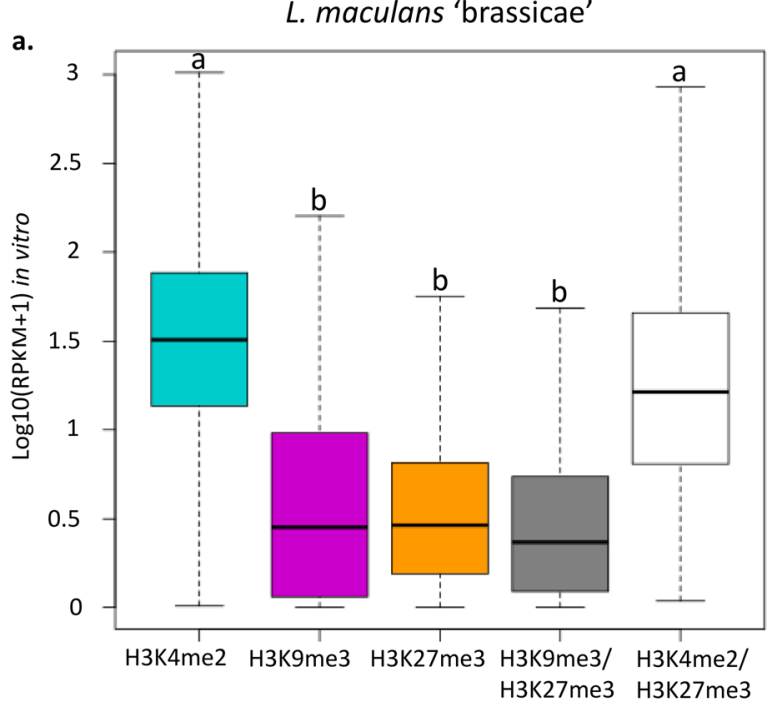

Fig. 6 Genes associated with heterochromatin are less expressed than genes associated with euchromatin in Leptosphaeria maculans 'brassicae' and Leptosphaeria maculans 'lepidii'. (a) L. maculans 'brassicae', Lmb; (b) L. maculans 'lepidii', Lml. Location of histone modifications in the genomes of $\mathrm{Lmb}$ and
2011). Blue: H3K4me2; purple: H3K9me3; orange: H3K27me3. Genes were considered as associated with any of the histone modifications when at least $1 \mathrm{bp}$ of the gene was found within the borders of the domain

Furthermore, among the orthologous genes, the genes associated with euchromatin in Lmb were also associated with euchromatin in Lml (as $83 \%$ of the genes conserved between the two species located in

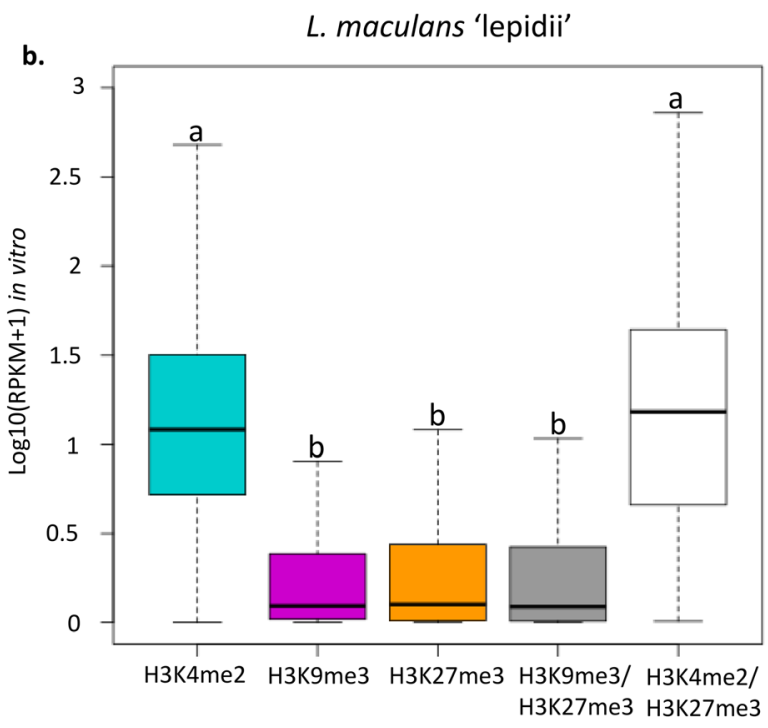

Lml was identified using RSEG (Song and Smith 2011), during axenic culture. RNA-seq was performed from $\mathrm{Lmb}$ or $\mathrm{Lml}$ grown one week in FRIES media. Blue: H3K4me2; purple: H3K9me3; orange: H3K27me3; grey: $\mathrm{H} 3 \mathrm{~K} 9 \mathrm{me} 3 / \mathrm{H} 3 \mathrm{~K} 27 \mathrm{me} 3$; white: H3K4me2/H3K27me3 
euchromatin in Lmb were also associated with euchromatin in Lml; Supplementary Table 10); half of the genes conserved between the two species and associated with H3K27me3 were also associated with H3K27me3 in Lml (Supplementary Table 10). On the contrary, conserved genes associated with heterochromatin in Lmb (either associated with $\mathrm{H} 3 \mathrm{~K} 9 \mathrm{me} 3$ or $\mathrm{H} 3 \mathrm{~K} 9 \mathrm{me} 3$ / $\mathrm{H} 3 \mathrm{~K} 27 \mathrm{me} 3$ ) were mostly found to be associated with $\mathrm{H} 3 \mathrm{~K} 27 \mathrm{me} 3$ in the Lml genome (48\% of the genes conserved between the two species associated with $\mathrm{H} 3 \mathrm{~K} 9 \mathrm{me} 3$ or H3K9me3/H3K27me3 were found associated with H3K27me3 in the Lml genome; Supplementary Table 10). No GO enrichment was found for these genes and predicted functions were sparse, with less than $45 \%$ of them having a functional annotation. Strikingly, the main enrichment was in genes encoding putative effectors (16\% of the genes).

Heterochromatin domains are enriched with proteinaceous and metabolic effector genes

We then focused on candidate proteinaceous or metabolic effectors and wondered whether they were conserved and showed a distinct pattern of histone modifications. In the genome of Lmb, 2466 genes (i.e. 19\% of the total genes) were associated with TE-rich regions (i.e. located within 2-kb distance of a TE sequence; Ohm et al. 2012), of which 289 genes encoded putative proteinaceous effectors. Hence, although a new prediction of the effector repertoire was performed here, based on the new assembly of the Lmb genome, TE-rich regions were significantly enriched with proteinaceous effector genes, as was already shown by Rouxel et al. (2011; $X^{2}$ test, $P=5.8 \cdot 10^{-10}$ ). Similarly, both H3K9me3- and H3K27me3-associated genes were significantly enriched with putative proteinaceous effectors, as they represented, respectively, $36 \%$ and $14 \%$ of the genes associated with these histone modifications in vitro $\left(X^{2}\right.$ test, $P<2.2 .10^{-16}$; Table 3 ; Supplementary Table 11). Likewise, in $\mathrm{Lml}$, TE-rich regions, H3K9me3 and H3K27me3 domains were significantly enriched with putative proteinaceous effector genes while $\mathrm{H} 3 \mathrm{~K} 4 \mathrm{me} 2$ domains were significantly depleted in such genes compared to the rest of the genome (Table 3; Supplementary Table 11). Among the 1080 putative proteinaceous effector genes predicted in $\mathrm{Lmb}$ ( $8.2 \%$ of the total predicted genes) and the 892 putative effector genes of $\mathrm{Lml}$ ( $7.9 \%$ of the total predicted genes), 274 were conserved between both species.
Hence, more than two-thirds of the effector repertoire is species specific $\left(X^{2}\right.$ test, $P<2.2 \cdot 10^{-16}$ ), confirming our previous findings (Grandaubert et al. 2014). Overall, orthologous effector-encoding genes were associated with the same types of chromatin domains in Lmb and Lml. For example, 98\% of the Lmb effector genes located in a euchromatin environment were also associated with $\mathrm{H} 3 \mathrm{~K} 4 \mathrm{me} 2$ in $\mathrm{Lml}$ ( $82 \%$ of $\mathrm{Lml}$ effector genes located in euchromatin regions were also associated with $\mathrm{H} 3 \mathrm{~K} 4 \mathrm{me} 2$ in Lmb). H3K27me3 and H3K9me3 domains were enriched with species-specific effector genes in both species (Table 3; Supplementary Table 11). As a striking example of the non-random location of effector genes in the two genomes, and the enrichment of heterochromatin regions with speciesspecific effectors, all currently known avirulence genes of Lmb were located in $\mathrm{H} 3 \mathrm{~K} 9 \mathrm{me} 3$ domains in vitro, consistent with the fact that these genes are located in TE-rich genomic compartments; none of the nine was conserved in the genome of $\mathrm{Lml}$. Twenty-eight other proteinaceous effector-encoding genes were located in TE-rich, H3K9me3 domains in $\mathrm{Lmb}$, and only one of them had a putative ortholog in Lml. The 274 orthologous genes encoding effectors were then investigated for their distribution in euchromatic/heterochromatic regions during vegetative growth and for conservation of their location between orthologs. There was no obvious bias in the distribution of chromatin marks among the 274 genes, which is comparable to the overall distribution of marks among all genes of the effector repertoires (data not shown). At the individual gene level, 83 genes were likewise associated with $\mathrm{H} 3 \mathrm{~K} 4 \mathrm{me} 2$ and 47 genes were likewise associated with H3K27me3 in both genomes.

The genomes of Lmb and Lml contain secondary metabolite gene clusters including key genes encoding PKS (polyketide synthases) and NRPS (non-ribosomal peptide synthetases). Twenty-seven such genes were predicted in Lmb among which 24 were conserved in $\mathrm{Lml}$, but they were overall absent from other closely related species (Rouxel et al. 2011; Grandaubert et al. 2014; Supplementary Table 12). Of these, only three have been experimentally demonstrated to be involved in Lmb pathogenicity, namely the PKS responsible for synthesis of abscisic acid (ABA), only expressed during cotyledon infection (Darma et al. 2019), the PKS responsible for producing phomenoic acid (Elliott et al. 2013) and the NRPS responsible for synthetizing 


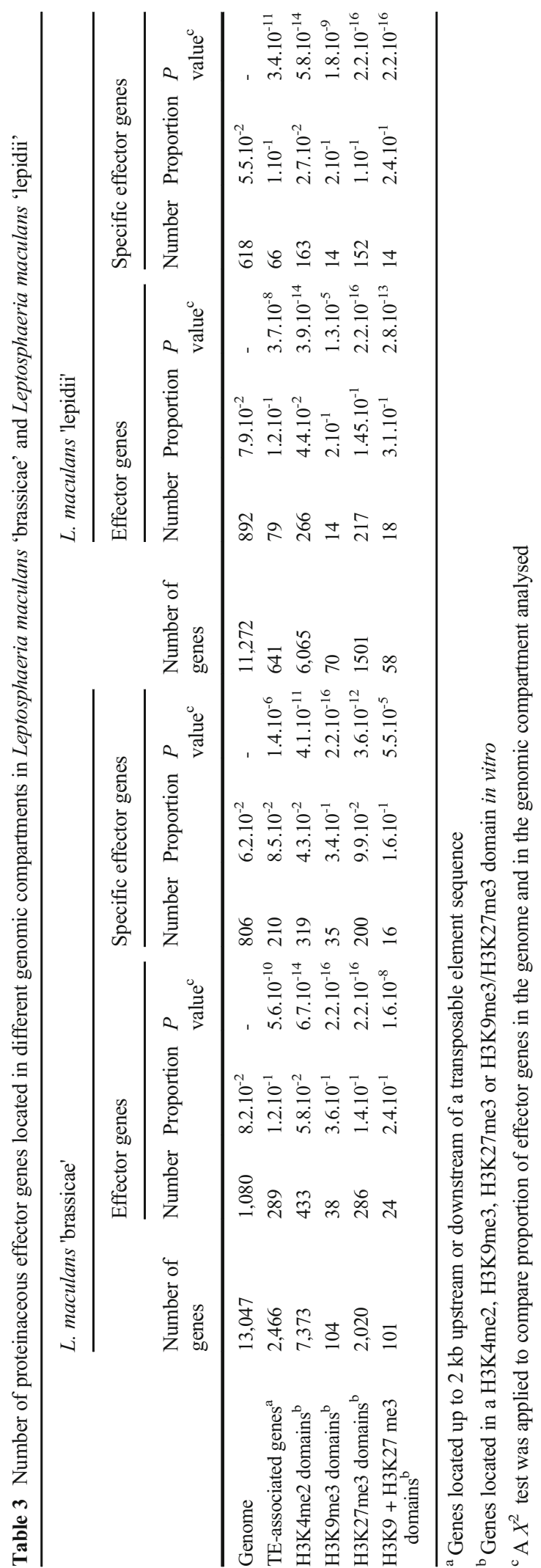

sirodesmin, a toxin produced during stem infection (Gardiner et al. 2004; Elliott et al. 2007). The ABA PKS and all seven genes of the cluster, which are intermingled with three TE-rich regions, were entirely absent from the genome of $\mathrm{Lml}$, while the other two were conserved (Supplementary Table 12). In Lmb, $81 \%$ of the PKS/NRPS-encoding genes were associated to H3K27me3 or H3K4me2/H3K27me3 domains (22 PKS/NRPS; Fisher's exact test, $P=1 \cdot 3 \cdot 10^{-7}$ ). Likewise in the Lml genome, H3K27me3 domains were enriched with PKS/NRPS-encoding genes (12 PKS/NRPS; Fisher's exact test, $P=1.7 .10^{-4}$ ), and all of them had orthologs associated with similar marks in Lmb. The similar chromatin context also resulted in similar regulation in most of the cases, with 19 of the orthologs being similarly expressed during in vitro growth (17 expressed and two repressed; Supplementary Table 12).

\section{Discussion}

The sister species L. maculans 'brassicae' and L. maculans 'lepidii' exhibit marked differences in their genome organization. Notably, Lmb has large TE-rich domains structuring the genome into alternating generich and TE-rich regions (Rouxel et al. 2011). In the genome of Lml, in contrast, TEs are evenly distributed across the genome and no compartmentalization of the genome is evident in relation to TE location (Grandaubert et al. 2014). The massive invasion of the Lmb genome by TEs occurred ca. 5 MYA and was postulated to have been instrumental in the separation of the two species (Grandaubert et al. 2014). This invasion may also have contributed to the rise of $\mathrm{Lmb}$ as a successful pathogen of $B$. napus due to the specific localization of a number of candidate effector genes in TE-rich regions of the genome (Rouxel et al. 2011; Grandaubert et al. 2014). Differences in genomic organization between these two closely related species could impact the underlying epigenomic landscape and have important consequences for fungal biology and pathogenicity. This could provide distinct strategies to regulate the expression of genes involved in stress response or pathogenicity, as it was shown in Lmb that histone modification $\mathrm{H} 3 \mathrm{~K} 9 \mathrm{me} 3$ is involved in the repression of avirulence genes during axenic growth (Soyer et al. 2014). To investigate whether different genomic organization impact the underlying epigenomic organization, we here compared the genome-wide location of 
three different histone modifications, typically associated with euchromatin or heterochromatin, in these two closely related phytopathogenic fungi, during axenic culture. We found that differences in the epigenomic landscape of $\mathrm{Lmb}$ and $\mathrm{Lml}$ are in accordance with their genome organization. In Lmb, very large $\mathrm{H} 3 \mathrm{~K} 9 \mathrm{me} 3$ domains are present, spanning large TE-rich regions, while such extremely large H3K9me3 domains are not observed in the Lml genome. Our findings corroborate previous epigenetic analyses of a few genes performed in vitro, pointing out that avirulence genes are located in heterochromatin (Soyer et al. 2014), but also demonstrate that heterochromatin (either associated with $\mathrm{H} 3 \mathrm{~K} 9 \mathrm{me} 3$ or $\mathrm{H} 3 \mathrm{~K} 27 \mathrm{me} 3$ ) is enriched with putative effector genes (Soyer et al. 2019).

Although Lmb and Lml have distinct genome organizations, they share a common distribution of histone modifications throughout their genome during axenic culture. Gene-rich regions are enriched with $\mathrm{H} 3 \mathrm{~K} 4 \mathrm{me} 2$ and $\mathrm{H} 3 \mathrm{~K} 27 \mathrm{me} 3$, while TE-rich regions are associated with $\mathrm{H} 3 \mathrm{~K} 9 \mathrm{me} 3$. The proportion of $\mathrm{H} 3 \mathrm{~K} 9 \mathrm{me} 3$ in a genome often reflects the TE content, as was described in Z. tritici or Podospora anserina (Schotanus et al. 2015; Carlier et al. 2020). In Lmb, having more than $30 \%$ of $\mathrm{TE}, 33 \%$ of the genome is associated with $\mathrm{H} 3 \mathrm{~K} 9 \mathrm{me} 3$ while the genome of $\mathrm{Lml}$, having a low TE content, shows a low enrichment in $\mathrm{H} 3 \mathrm{~K} 9 \mathrm{me} 3$ (4\% of H3K9me3). Domains enriched with H3K4me2 and domains enriched with $\mathrm{H} 3 \mathrm{~K} 9 \mathrm{me} 3$ are mutually exclusive in the genomes of $\mathrm{Lmb}$ and $\mathrm{Lml}$, as was shown in Neurospora crassa, Fusarium fujikuroi, Z. tritici or P. anserina (Smith et al. 2008; Jamieson et al. 2013; Wiemann et al. 2013; Schotanus et al. 2015; Carlier et al. 2020). H3K27me3 has been detected in most filamentous fungi investigated so far, except in Mucor, Rhizopus or Aspergilli such as Aspergillus nidulans (Gacek-Matthews et al. 2016; reviewed in Freitag 2017). In sub-telomeric regions of Lmb, H3K9me3 and $\mathrm{H} 3 \mathrm{~K} 27 \mathrm{me} 3$ domains overlap over repetitive sequences, which is also the case in $N$. crassa or Z. tritici (Smith et al. 2008; Jamieson et al. 2013; Jamieson et al. 2018; Schotanus et al. 2015). In the genome of Lmb, TE-rich regions are enriched with $\mathrm{H} 3 \mathrm{~K} 9 \mathrm{me} 3$ but, except for the sub-telomeric regions, no enrichment in H3K27me3 was associated with TEs on chromosomal arms. This contrasts with the organization of TE-rich regions in Z. tritici, which are enriched with both heterochromatin modifications (Schotanus et al. 2015). The situation in Lmb is also different from that of Fusarium oxysporum in which most TE sequences are embedded in $\mathrm{H} 3 \mathrm{~K} 27 \mathrm{me} 3$ domains (Fokkens et al. 2018). We confirmed that, as in most eukaryotes, H3K4me2 domains are associated with expressed genes while $\mathrm{H} 3 \mathrm{~K} 9 \mathrm{me} 3$ and $\mathrm{H} 3 \mathrm{~K} 27 \mathrm{me} 3$ domains are associated with silent genes. In Lmb, although the locations of $\mathrm{H} 3 \mathrm{~K} 4 \mathrm{me} 2$ and $\mathrm{H} 3 \mathrm{~K} 27 \mathrm{me} 3$ domains do not overlap at a genome-wide scale, more than 2000 genes were found to be associated with both histone methylations. This is strikingly different to $\mathrm{Lml}$, where only 300 genes were associated with both histone modifications, and Z. tritici, where 400 genes are embedded in such domains (Schotanus et al. 2015; Soyer et al. 2019). Bivalent domains are defined as chromatin regions associated with both repressive and permissive histone modifications (Bernstein et al. 2006). The large number of genes associated with $\mathrm{H} 3 \mathrm{~K} 27 \mathrm{me} 3$ and $\mathrm{H} 3 \mathrm{~K} 4 \mathrm{me} 2$ during axenic culture in Lmb might indicate a biological specificity of this species and the existence of bivalent domains, because these two modifications have antagonistic effects on gene expression (Harikumar and Meshorer 2015). Altogether, our findings show that, despite differences in genomic organization between $\mathrm{Lmb}$ and $\mathrm{Lml}$ (and the other fungi in which epigenomic analyses were so far performed), the epigenomic landscape is overall conserved.

While $\mathrm{H} 3 \mathrm{~K} 9 \mathrm{me} 3$ and $\mathrm{H} 3 \mathrm{~K} 27 \mathrm{me} 3$ are signatures of heterochromatin, $\mathrm{H} 3 \mathrm{~K} 9 \mathrm{me} 3$ is considered to be typical of constitutive heterochromatin, being associated with repeats and involved in genome stability, whereas $\mathrm{H} 3 \mathrm{~K} 27 \mathrm{me} 3$ is considered to be associated with facultative heterochromatin that is easily reversed to a euchromatin state under certain abiotic and biotic stress conditions (Grewal and Jia 2007). However, dogmas regarding conventional definitions of facultative or constitutive heterochromatin seem to be challenged in fungi, or at least in the few plant pathogenic fungi in which epigenomic analyses have been performed. While the co-localization of H3K9me3 and TEs in the Lmb and $\mathrm{Lml}$ genomes is consistent with a constitutive heterochromatin state, the fact that $\mathrm{H} 3 \mathrm{~K} 9 \mathrm{me} 3$ domains encompass genes that are expressed during interaction with oilseed rape suggests that these regions may correspond to a category of facultative heterochromatin in Lmb. Genes associated with $\mathrm{H} 3 \mathrm{~K} 9 \mathrm{me} 3$ are almost all located in the middle of repeated elements, in sub-telomeric areas, or very close to the edge of regions enriched with repeated elements. Moreover, some of these genes, including the nine cloned avirulence genes of $\mathrm{Lmb}$ which 
are located within AT-rich isochores (e.g. AvrLml or AvLm6; Gout et al. 2006; Fudal et al. 2007) including sub-telomeric regions (e.g. AvrLm3 or AvrLm10; Plissonneau et al. 2016; Petit-Houdenot et al. 2019), are highly transcribed upon host infection (Rouxel et al. 2011). This finding, together with other studies, questions the 'constitutive' nature of heterochromatin associated with $\mathrm{H} 3 \mathrm{~K} 9 \mathrm{me} 3$. In contrast, the location of H3K27me3 in the genomes of Lmb and Lml supports its association with facultative heterochromatin. Indeed, we found H3K27me3 associated with coding sequences and enriched with genes encoding proteinaceous and metabolic effectors or proteins involved in stress responses. Most of these genes are silenced during vegetative growth but induced during plant infection. In fungi, recent analyses also highlighted a role for $\mathrm{H} 3 \mathrm{~K} 27 \mathrm{me} 3$ in genome organization and stability. For instance, the dispensable chromosomes of $Z$. tritici are twice as rich in TEs as the core chromosomes, and whereas there is no significant enrichment in $\mathrm{H} 3 \mathrm{~K} 9 \mathrm{me} 3$ on the dispensable chromosomes, they are entirely covered by H3K27me3 (Schotanus et al. 2015). In Z. tritici, the loss of H3K27me3 was found to increase the stability of some accessory chromosomes (Möller et al. 2019). In Z. tritici and $N$. crassa, H3K27me3 is relocated towards normal constitutive heterochromatin (i.e. H3K9me3 domains) under genotoxic stress, such as the loss of $\mathrm{H} 3 \mathrm{~K} 9 \mathrm{me} 3$ after inactivation of KMT1 (Basenko et al. 2015; Möller et al. 2019). Altogether, these findings suggest that although $\mathrm{H} 3 \mathrm{~K} 27 \mathrm{me} 3$ is an important regulator of gene expression involved in development or response to various stresses, it also plays a role in the maintenance of genome integrity. In Lmb and Lml, no particular association of H3K27me3 with TEs was identified and the dispensable chromosome of $\mathrm{Lmb}$ is not enriched with $\mathrm{H} 3 \mathrm{~K} 27 \mathrm{me}$. The only association of H3K27me3 with constitutive chromatin was at the chromosome ends of $\mathrm{Lmb}$, where we found overlaps between $\mathrm{H} 3 \mathrm{~K} 9 \mathrm{me} 3$ and H3K27me3. Inactivation of KMT1 and KMT6 would help investigate whether H3K27me3 could also be involved in genome stability and relocation of heterochromatin marks in Lmb and Lml.

The heterochromatin domains of Lmb and Lml are rich in species-specific genes, genes involved in stress responses and putative proteinaceous or metabolic effectors. This supports the view that chromatin remodelling mechanisms are an efficient way to rapidly modulate gene expression under stress conditions or during biotic interactions, although the dynamics of chromatin structure might not be the sole regulator of these complex biological processes (Soyer et al. 2015a; Tan and Oliver 2017). This pattern is conserved in other plant-associated fungi, independently of their mode of interaction with their host (Ma et al. 2010; Rouxel et al. 2011; Chujo and Scott 2014; Schotanus et al. 2015; Faino et al. 2016; Vlaardingerbroek et al. 2016; Dallery et al. 2017; Krishnan et al. 2018; Soyer et al. 2019). Even outside of TE-rich regions, heterochromatin is often found enriched with pathogenicity-related genes, whether they encode proteinaceous effectors or are involved in the production of secondary metabolites (e.g. Connolly et al. 2013; Wiemann et al. 2013; Chujo and Scott 2014; Schotanus et al. 2015; Fokkens et al. 2018; Soyer et al. 2019). Importantly, sets of genes upregulated during host infection are found associated with heterochromatin in vitro (Fokkens et al. 2018; Haueisen et al. 2018; Soyer et al. 2019). One of our initial postulates was that invasion of the L. maculans genome by TEs contributed to the rise of $L m b$ as a pathogen specialized on Brassicas with greater pathogenic abilities than the non-invaded sister species. While $\mathrm{Lml}$ has never been found to be able to infect $B$. napus under our experimental conditions or isolated from our experimental fields (M-H. Balesdent \& T. Rouxel, unpublished data), previous work reported its ability to infect other crucifers (Petrie 1969). Its isolation from ascocarps on stems of Lepidium sp. also suggests its infection strategy is similar to that of Lmb on B. napus. So far, all avirulence genes of $\mathrm{Lmb}$, which can be recognized by the plant immune system to set up defence reactions, are located in TE-rich regions and associated with $\mathrm{H} 3 \mathrm{~K} 9 \mathrm{me} 3$. In contrast, in both species, we found conserved putative effector genes (either proteinaceous or metabolic) harboured in similar H3K27me3 heterochromatin environments. In Lmb, some of them are expressed during stem infection of oilseed rape and have been named 'late' effector genes (Gervais et al. 2017). The contrasting heterochromatic environment (H3K9me3 vs. H3K27me3) for avirulence genes and 'late' effector genes might reflect a very different adaptive behaviour because effector genes that are expressed early, and likely to be 'recognized' by the plant surveillance machinery at the onset of penetration, are also those which are subject to accelerated evolution under selection (Rouxel et al. 2011). This suggests that there may be a selective advantage for the fungus to partition genes more likely to be recognized by the host plant within H3K9me3 domains. Nevertheless, the location of putative pathogenicity genes, including 
orthologues, in similar heterochromatin regions in the genomes of Lmb and Lml suggests that basic pathogenicity programs are independent of genome invasion by TEs, and points to the likewise importance of chromatin context on transcriptome shaping during infection. Taken together, these data support our previous hypothesis that the localization of effector genes in plastic genomic compartments is an efficient way to regulate the expression of sets of genes scattered throughout the genome that are involved in similar biological processes (Soyer et al. 2015a).

\section{Conclusions}

To the best of our knowledge, previous comparative analyses of histone modifications have been performed in model organisms such as mouse (Mikkelsen et al. 2010) but none considered multiple histone modifications (typical for both heterochromatin and euchromatin) in closely related phytopathogenic fungi. So far, little is known about the extent of conservation of histone modifications among closely related species and how this may influence pathogenesis. Comparative genomics has allowed analyses of gene evolution (notably proteinaceous effectors), the location of effector genes in genomes or the diversity of effector repertoires in relation to host specialization or fungal lifestyles. The role of the epigenome is increasingly recognized in plant pathogenic fungi as an important regulator of genome structure (e.g. Basenko et al. 2015; Möller et al. 2019, 2020) and the expression of genes encoding effectors (e.g. Chujo and Scott 2014; Soyer et al. 2014, 2019; Meile et al. 2020). The next step will be to exploit epigenomic data in a comparative framework to better understand the role of the epigenomic landscape in adaptation of pathogens to environmental changes, modulation of interactions with the holobiont, host adaptation and specialization.

Supplementary Information The online version contains supplementary material available at https://doi.org/10.1007/s10577021-09658-1.

Acknowledgements Authors wish to thank all members of the 'Effectors and Pathogenesis of L. maculans' and 'Environmental Genomics' groups; M. Freitag and J. Grandaubert for fruitful discussions; and R. O'Connell for the English proofreading of this paper.
Author contributions Conceived, initiated and coordinated the project: JLS, TR, EHS, IF. Designed the experiments: JLS, EHS, IF. Performed the experiments: JLS. Analysis of the data: JLS, CC, EJG, NL. Interpretation of the data: JLS, TR, EHS, IF. Writing of the draft: JLS. Editing of the draft: JLS, CC, TR, EHS, IF.

Funding J.L. Soyer was funded by a 'Contrat Jeune Scientifique' grant from INRA. The 'Effectors and Pathogenesis of L. maculans' group benefits from the support of Saclay Plant Sciences-SPS (ANR-17-EUR-0007). Research on fungal histone modifications in the group of E.H. Stukenbrock is funded by the Max Planck Society, Germany and in the group of J.L. Soyer and I. Fudal by the French National Research Agency project ChOPIN (ANR-19-CE20-0022). The funders had no role in study design, data collection and interpretation or the decision to submit the work for publication.Data availabilityOmic data are available under the GEO accession number GSE150127.

\section{Declarations}

Ethics approval and consent to participate Not applicable.

Consent for publication Not applicable.

Conflict of interest The authors declare no competing interests.

Open Access This article is licensed under a Creative Commons Attribution 4.0 International License, which permits use, sharing, adaptation, distribution and reproduction in any medium or format, as long as you give appropriate credit to the original author(s) and the source, provide a link to the Creative Commons licence, and indicate if changes were made. The images or other third party material in this article are included in the article's Creative Commons licence, unless indicated otherwise in a credit line to the material. If material is not included in the article's Creative Commons licence and your intended use is not permitted by statutory regulation or exceeds the permitted use, you will need to obtain permission directly from the copyright holder. To view a copy of this licence, visit http://creativecommons.org/licenses/by/4.0/.

\section{References}

Almagro Armenteros JJ, Salvatore M, Emanuelsson O et al (2019) Detecting sequence signals in targeting peptides using deep learning. Life Sci Alliance 2:5

Ansan-Melayah D, Balesdent M-H, Buee M et al (1995) Genetic characterization of $A v r L m 1$, the first avirulence gene of Leptosphaeria maculans. Phytopathology 85:1525-1529

Balesdent M-H, Fudal I, Ollivier B (2013) The dispensable chromosome of Leptosphaeria maculans shelters an effector gene conferring avirulence towards Brassica rapa. New Phytol 198:887-898 
Basenko EY, Sasaki T, Ji L et al (2015) Genome-wide redistribution of H3K27me3 is linked to genotoxic stress and defective growth. Proc Natl Acad Sci U S A 112:E6339-E6348

Bernstein BE, Mikkelsen TS, Xie X et al (2006) A bivalent chromatin structure marks key developmental genes in embryonic stem cells. Cell 125:315-326

Bolger AM, Lohse M, Usadel B (2014) Trimmomatic: a flexible trimmer for Illumina sequence data. Bioinformatics 30:2114 2120

Carlier F, Debuchy R, Maroc L, et al (2020) Loss of Polycomb Protein EZH2 causes major depletion of H3K27 and H3K9 tri-methylation and developmental defects in the fungus Podospora anserina. Preprint at bioRxiv

Chujo T, Scott B (2014) Histone H3K9 and H3K27 methylation regulates fungal alkaloid biosynthesis in a fungal endophyteplant symbiosis: K9 and K27 methylation regulates symbiosis. Mol Microbiol 92:413-434

Chuma I, Isobe C, Hotta Y et al (2011) Multiple translocation of the AVR-Pita effector gene among chromosomes of the rice blast fungus Magnaporthe oryzae and related species. PLoS Pathog 7:e1002147

Collemare J, O'Connell R, Lebrun M-H (2019) Nonproteinaceous effectors: the terra incognita of plant-fungal interactions. New Phytol 223:590-596

Connolly LR, Smith KM, Freitag M (2013) The Fusarium graminearum histone H3K27 methyltransferase KMT6 regulates development and expression of secondary metabolite gene clusters. PLoS Genet 9:e1003916

Croll D, McDonald BA (2012) The accessory genome as a cradle for adaptive evolution in pathogens. PLoS Pathog 8: e1002608

Dallery JF, Lapalu N, Zampounis A et al (2017) Gapless genome assembly of Colletotrichum higginsianum reveals chromosome structure and association of transposable elements with secondary metabolite gene clusters. BMC Genomics 18:667

Darma R, Lutz A, Elliott CE, Idnurm A (2019) Identification of a gene cluster for the synthesis of the plant hormone abscisic acid in the plant pathogen Leptosphaeria maculans. Fungal Genet Biol 130:62-71

Daverdin G, Rouxel T, Gout L et al (2012) Genome structure and reproductive behaviour influence the evolutionary potential of a fungal phytopathogen. PLoS Pathog 8:1003020

Dobin A, Gingeras TR (2015) Mapping RNA-seq reads with STAR. Curr Protoc Bioinformatics 3(51):11.14.1-11.14.19

Dutreux F, Da Silva C, d'Agata L et al (2018) De novo assembly and annotation of three Leptosphaeria genomes using Oxford Nanopore MinION sequencing. Sci Data 5:180235

Elliott CE, Gardiner DM, Thomas G et al (2007) Production of the toxin sirodesmin PL by Leptosphaeria maculans during infection of Brassica napus. Mol Plant Pathol 8:791-802

Elliott CE, Callahan DL, Schwenk D et al (2013) A gene cluster responsible for biosynthesis of phomenoic acid in the plant pathogenic fungus, Leptosphaeria maculans. Fungal Genet Biol 53:50-58

Emms DM, Kelly S (2015) OrthoFinder: solving fundamental biases in whole genome comparisons dramatically improves orthogroup inference accuracy. Genome Biol 16:157

Faino L, Seidl MF, Shi-Kunne X et al (2016) Transposons passively and actively contribute to evolution of the two-speed genome of a fungal pathogen. Genome Res 26:1091-1100
Feurtey A, Lorrain C, Croll D, et al (2019) Genome compartmentalization predates species divergence in the plant pathogen genus Zymoseptoria. Preprint at bioRXiv

Fisher MC, Hawkins NJ, Sanglard D, Gurr SJ (2018) Worldwide emergence of resistance to antifungal drugs challenges human health and food security. Science 360:739-742

Fokkens L, Shahi S, Connolly LR, et al (2018) The multi-speed genome of Fusarium oxysporum reveals association of histone modifications with sequence divergence and footprints of past horizontal chromosome transfer events. Preprint at bioRXiv

Freitag M (2017) Histone methylation by SET domain proteins in Fungi. Annu Rev Microbiol 71:413-439

Fudal I, Ross S, Gout L et al (2007) Heterochromatin-like regions as ecological niches for avirulence genes in the Leptosphaeria maculans genome: map-based cloning of AvrLm6. Mol Plant-Microbe Interact 20:459-470

Gacek-Matthews A, Berger H, Sasaki T et al (2016) KdmB, a Jumonji Histone H3 demethylase, regulates genome-wide $\mathrm{H} 3 \mathrm{~K} 4$ trimethylation and is required for normal induction of secondary metabolism in Aspergillus nidulans. PLoS Genet 12:e1006222

Gardiner DM, Cozijnsen AJ, Wilson LM et al (2004) The sirodesmin biosynthetic gene cluster of the plant pathogenic fungus Leptosphaeria maculans. Mol Microbiol 53:13071318

Gervais J, Plissonneau C, Linglin J et al (2017) Different waves of effector genes with contrasted genomic location are expressed by Leptosphaeria maculans during cotyledon and stem colonization of oilseed rape. Mol Plant Pathol 18: 1113-1126

Ghanbarnia K, Fudal I, Larkan NJ et al (2015) Rapid identification of the Leptosphaeria maculans Avirulence gene AvrLm2 using an intraspecific comparative genomics approach. Mol Plant Pathol 16:699-709

Ghanbarnia K, Ma L, Larkan NJ et al (2018) Leptosphaeria maculans AvrLm9: a new player in the game of hide and seek with AvrLm4-7. Mol Plant Pathol 19:1754-1764

Gout L, Fudal I, Kuhn M-L et al (2006) Lost in the middle of nowhere: the $A v r L m 1$ avirulence gene of the Dothideomycete Leptosphaeria maculans. Mol Microbiol 60:67-80

Grandaubert J, Lowe RG, Soyer JL et al (2014) Transposable element-assisted evolution and adaptation to host plant within the Leptosphaeria maculans-Leptosphaeria biglobosa species complex of fungal pathogens. BMC Genomics 15: 891

Grewal SI, Jia S (2007) Heterochromatin revisited. Nat Rev Genet 8:35-46

Harikumar A, Meshorer E (2015) Chromatin remodeling and bivalent histone modifications in embryonic stem cells. EMBO Rep 16:1609-1619

Haueisen J, Möller M, Eschenbrenner CJ et al (2018) Highly flexible infection programs in a specialized wheat pathogen. Ecol Evol 9:275-294

Jamieson K, Rountree MR, Lewis ZA et al (2013) Regional control of histone H3 lysine 27 methylation in Neurospora. Proc Natl Acad Sci U S A 110:6027-6032

Jamieson K, McNaught KJ, Ormsby T et al (2018) Telomere repeats induce domains of $\mathrm{H} 3 \mathrm{~K} 27$ methylation in Neurospora. Elife 7:e31216 
Jones JD, Dangl JL (2006) The plant immune system. Nature 444: 323-329

de Jonge R, Bolton MD, Kombrink A et al (2013) Extensive chromosomal reshuffling drives evolution of virulence in an asexual pathogen. Genome Res 23:1271-1282

Kim HY (2017) Statistical notes for clinical researchers: Chisquared test and Fisher's exact test. Restor Dent Endod 42: $152-155$

Krishnan P, Meile L, Plissonneau C (2018) Transposable element insertions shape gene regulation and melanin production in a fungal pathogen of wheat. BMC Biol 16:78

Langmead B, Salzberg SL (2012) Fast gapped-read alignment with Bowtie 2. Nat Methods 9:357-359

Leclair S, Ansan-Melayah D, Rouxel T et al (1996) Meiotic behaviour of the minichromosome in the phytopathogenic ascomycete Leptosphaeria maculans. Curr Genet 30:541548

Lo Presti L, Lanver D, Schweizer G et al (2015) Fungal effectors and plant susceptibility. Annu Rev Plant Biol 66:513-545

Lukito Y, Lee K, Noorifar N, et al (2020) Host infection by the grass-symbiotic fungus Epichloë festucae requires catalytically active $\mathrm{H} 3 \mathrm{~K} 9$ and $\mathrm{H} 3 \mathrm{~K} 36$ methyltransferases. Preprint at bioRxiv. https://doi.org/10.1101/2020.09.01.277384

Ma LJ, van der Does HC, Borkovich KA et al (2010) Comparative genomics reveals mobile pathogenicity chromosomes in Fusarium. Nature 464:367-373

Meile L, Peter J, Puccetti G et al (2020) Chromatin dynamics contribute to the spatiotemporal expression pattern of virulence genes in a fungal plant pathogen. mBio 11:e02343e02320

Mikkelsen TS, Ku M, Jaffe DB et al (2007) Genome-wide maps of chromatin state in pluripotent and lineage-committed cells. Nature 448:553-560

Mikkelsen TS, Xu Z, Zhang X (2010) Comparative epigenomic analysis of murine and human adipogenesis. Cell 143:156169

Möller M, Stukenbrock EH (2017) Evolution and genome architecture in fungal plant pathogens. Nat Rev Microbiol 15:771

Möller M, Schotanus K, Soyer JL et al (2019) Destabilization of chromosome structure by histone H3 lysine 27 methylation. PLoS Genet 15:e1008093

Möller M, Habig M, Lorrain C, et al (2020) Recent loss of the Dim2 cytosine DNA methyltransferase impacts mutation rate and evolution in a fungal plant pathogen. Preprint at bioRXiv. https://doi.org/10.1101/2020.03.27.012203

Neik TX, Ghanbarnia K, Ollivier B, et al (2020) Two independent approaches converge to the cloning of a new Leptosphaeria maculans avirulence effector gene, AvrLmS-Lep2. Preprint at bioRXiv. https://doi.org/10.1101/2020.10.02.322479

Ohm RA, Feau N, Henrissat B et al (2012) Diverse lifestyles and strategies of plant pathogenesis encoded in the genomes of eighteen Dothideomycetes fungi. PLoS Pathog 8:e1003037

Parlange F, Daverdin G, Fudal I et al (2009) Leptosphaeria maculans avirulence gene AvrLm4-7 confers a dual recognition specificity by the $R \operatorname{lm} 4$ and $R \operatorname{lm} 7$ resistance genes of oilseed rape, and circumvents Rlm4-mediated recognition through a single amino acid change. Mol Microbiol 71: 851-863

Petersen TN, Brunak S, von Heijne G et al (2011) SignalP 4.0: discriminating signal peptides from transmembrane regions. Nat Methods 8:785-786
Petit-Houdenot Y, Degrave A, Meyer M et al (2019) A two genes for - one gene interaction between Leptosphaeria maculans and Brassica napus. New Phytol 223:397-411

Petrie GA (1969) Variability in Leptosphaeria maculans (Desm.) Ces. et De Not., the cause of blackleg of rape. $\mathrm{PhD}$ thesis, University of Saskatchewan (215 pp.)

Plissonneau C, Daverdin G, Ollivier B et al (2016) A game of hide and seek between avirulence genes AvrLm4-7 and AvrLm3 in Leptosphaeria maculans. New Phytol 209:1613-1624

Plissonneau C, Rouxel T, Chèvre A-M et al (2018) One gene-one name: the AvrLmJ1 avirulence gene of Leptosphaeria maculans is AvrLm5. Mol Plant Pathol 19:1012-1016

Robinson MD, McCarthy DJ, Smyth GK (2010) edgeR: a Bioconductor package for differential expression analysis of digital gene expression data. Bioinformatics 26:139-140

Rocafort M, Fudal I, Mesarich CH (2020) Apoplastic effector proteins of plant-associated fungi and oomycetes. Curr Opin Plant Biol 56:9-19

Rouxel T, Balesdent MH (2005) The stem canker (blackleg) fungus, Leptosphaeria maculans, enters the genomic era. Mol Plant Pathol 6:225-241

Rouxel T, Balesdent MH (2017) Life, death and rebirth of avirulence effectors in a fungal pathogen of Brassica crops, Leptosphaeria maculans. New Phytol 214:526-532

Rouxel T, Grandaubert J, Hane JK et al (2011) Effector diversification within compartments of the Leptosphaeria maculans genome affected by Repeat-Induced Point mutations. Nat Commun 2:202

Sánchez-Vallet A, Fouché S, Fudal I et al (2018) The genome biology of effector gene evolution in filamentous plant pathogens. Annu Rev Phytopathol 56:21-40

Schotanus K, Soyer JL, Connolly LR et al (2015) Histone modifications rather than the novel regional centromeres of Zymoseptoria tritici distinguish core and accessory chromosomes. Epigenetics Chromatin 8:41

Seidl MF, Thomma BPHJ (2017) Transposable elements direct the coevolution between plants and microbes. Trends Genet 33: $842-851$

Shannon P, Markiel A, Ozier O et al (2003) Cytoscape: a software environment for integrated models of biomolecular interaction networks. Genome Res 13:2498-2504

Smith KM, Kothe GO, Matsen CB et al (2008) The fungus Neurospora crassa displays telomeric silencing mediated by multiple sirtuins and by methylation of histone $\mathrm{H} 3$ lysine 9. Epigenetics Chromatin 1:5

Song Q, Smith AD (2011) Identifying dispersed epigenomic domains from ChIP-Seq data. Bioinformatics 27:870-871

Soyer JL, El Ghalid M, Glaser N et al (2014) Epigenetic control of effector gene expression in the plant pathogenic fungus Leptosphaeria maculans. PLoS Genet 10:e1004227

Soyer JL, Rouxel T, Fudal I (2015a) Chromatin-based control of effector gene expression in plant-associated fungi. Curr Opin Plant Biol 26:51-56

Soyer JL, Möller M, Schotanus K et al (2015) Chromatin analyses of Zymoseptoria tritici: Methods for chromatin immunoprecipitation followed by high-throughput sequencing (ChIPseq). Fungal Genet Biol 79:63-70

Soyer JL, Grandaubert J, Haueisen J et al (2019) In eplanta chromatin immunoprecipitation in Zymoseptoria tritici reveals chromatin-based regulation of putative effector gene expression. Preprint at bioRxiv 
Sperschneider J, Gardiner DM, Dodds PN et al (2016) EffectorP: predicting fungal effector proteins from secretomes using machine learning. New Phytol 210:743-761

Stergiopoulos I, De Kock MJ, Lindhout P et al (2007) Allelic variation in the effector genes of the tomato pathogen Cladosporium fulvum reveals different modes of adaptive evolution. Mol Plant-Microbe Interact 20:1271-1283

Stukenbrock EH, Bataillon T, Dutheil JY et al (2011) The making of a new pathogen: insights from comparative population genomics of the domesticated wheat pathogen Mycosphaerella graminicola and its wild sister species. Genome Res 21:2157-2166

Tan KC, Oliver RP (2017) Regulation of proteinaceous effector expression in phytopathogenic fungi. PLoS Pathog 13: e1006241

Thorvaldsdóttir H, Robinson JT, Mesirov JP (2013) Integrative Genomics Viewer (IGV): high-performance genomics data visualization and exploration. Brief Bioinform 14:178-192
Van de Wouw AP, Lowe RG, Elliott CE et al (2014) An avirulence gene, AvrLmJ1, from the blackleg fungus, Leptosphaeria maculans, confers avirulence to Brassica juncea cultivars. Mol Plant Pathol 15:523-530

Vlaardingerbroek I, Beerens B, Schmidt SM et al (2016) Dispensable chromosomes in Fusarium oxysporum f. sp. lycopersici. Mol Plant Pathol 17:1455-1466

Wiemann P, Sieber CM, von Bargen KW et al (2013) Deciphering the cryptic genome: genome-wide analyses of the rice pathogen Fusarium fujikuroi reveal complex regulation of secondary metabolism and novel metabolites. PLoS Pathog 9: e1003475

Zhang Z, Wen J, Li J et al (2018) The evolution of genomic and epigenomic features in two Pleurotus fungi. Sci Rep 8:8313

Publisher's note Springer Nature remains neutral with regard to jurisdictional claims in published maps and institutional affiliations. 